\title{
Weiterarbeit nach Erreichen des Pensionsalters - Auswirkungen auf Ihre Versicherungen und Ihre Vorsorge
}

Thomas Roth, Roger Ledermann

Korrespondenz: Thomas Roth Roth Gygax \& Partner AG FMH Insurance Services Moosstrasse 2

CH-3073 Gümligen Tel. 0319595000 Fax 0319595010

thomas.roth@fmhinsurance.ch
Viele Ärztinnen und Ärzte nutzen heute die Möglichkeiten einer flexiblen Pensionierung. Während einige bereits ein paar Jahre vor dem ordentlichen Pensionsalter in den Ruhestand treten, arbeiten andere auch nach Erreichen des 64. beziehungsweise 65. Altersjahrs noch weiter. Dies hat erheblichen Einfluss auf die Vorsorge- und Versicherungssituation. Im folgenden finden sich die wichtigsten Punkte, die es zu beachten gilt.

\section{Berufshaftpflichtversicherung, Praxis-Sachversicherung, \\ Berufsrechtsschutzversicherung}

Diese Versicherungen laufen normalerweise automatisch mit dem gleichen Deckungsumfang weiter.

\section{Taggeldversicherung}

Je nach Vertrag läuft diese Versicherung mit Erreichen des Pensionsalters ab. Bei guten Lösungen kann ein Taggeld bis zu 5 Jahren weiterversichert werden. Jedoch sind nur noch maximal 180 Taggelder versicherbar.

\section{Personalversicherungen}

Die Versicherungen für Angestellte (BVG, Krankentaggeld, UVG) laufen automatisch weiter.

\section{Krankenkasse}

Sofern vor Erreichen des 65. Altersjahres eine separate Unfalldeckung bestand (UVG) und das Unfallrisiko in der Krankenkasse ausgeschlossen war, muss dieses nun wieder eingeschlossen werden.

\section{Berufliche Vorsorge BVG}

Die Pensionskasse kann bei einigen Stiftungen freiwillig bis zu 5 Jahren weitergeführt werden. Je nach Kasse gibt es spezielle Vorsorgepläne mit reduzierten Leistungen im Todesfall oder bei Erwerbsunfähigkeit. Problematisch sind jedoch Einkäufe nach dem ordentlichen Pensionsalter, da diese meistens von der Steuerverwaltung nicht akzeptiert werden. Es lohnt sich daher, Einkäufe bereits vorher zu tätigen, insbesondere, da es für die Kapitaloption eine 3-jährige Sperrfrist auf die getätigten Einkäufe gibt.
Freizügigkeitskonti können ebenfalls weitergeführt werden und müssen spätestens 5 Jahre nach dem ordentlichen Pensionsdatum bezogen werden.

\section{Säule 3a}

Die gebundene Vorsorge kann maximal 5 Jahre über das ordentliche Pensionsalter weitergeführt werden. Dabei muss jährlich eine Bestätigung über die Erwerbstätigkeit vorgelegt werden.

\section{Private Risikoversicherungen}

Private Erwerbsunfähigkeitsversicherungen können nur bis zum ordentlichen Pensionsalter abgeschlossen werden und enden mit Erreichen dieses Alters automatisch. Todesfallversicherungen können auch über das Pensionierungsalter vereinbart werden. Je nach Gesellschaft muss jedoch der Bedarf belegt werden (z. B. Absicherung einer Hypothek).

\section{AHV}

Die Leistungen der AHV können um bis zu 5 Jahre aufgeschoben werden, wobei die Leistungen entsprechend erhöht werden (Tab. 1). Es muss dazu vor Erreichen des ordentlichen Pensionsalters eine schriftliche Erklärung bei der zuständigen Ausgleichskasse eingereicht werden.

Es gibt also viele Punkte, die beachtet werden müssen. Es lohnt sich daher, frühzeitig die richtigen Weichen zu stellen.

Tabelle 1

Erhöhung der AHV-Rente in Prozent bei einem Aufschub.

\begin{tabular}{|lcccc} 
& $\begin{array}{l}\mathbf{0 - 2} \\
\text { Monate }\end{array}$ & $\begin{array}{l}\text { 3-5 } \\
\text { Monate }\end{array}$ & $\begin{array}{l}\text { 6-8 } \\
\text { Monate }\end{array}$ & $\begin{array}{l}\text { 9-11 } \\
\text { Monate }\end{array}$ \\
\hline 1 Jahr & $5,2 \%$ & $6,6 \%$ & $8,0 \%$ & $9,4 \%$ \\
\hline 2 Jahre & $10,8 \%$ & $12,3 \%$ & $13,9 \%$ & $15,5 \%$ \\
\hline 3 Jahre & $17,1 \%$ & $18,8 \%$ & $20,5 \%$ & $22,2 \%$ \\
\hline 4 Jahre & $24,0 \%$ & $25,8 \%$ & $27,7 \%$ & $29,6 \%$ \\
\hline 5 Jahre & $31,5 \%$ & & & \\
\hline
\end{tabular}

